\title{
Carbon Nanotubes/Magnetite Hybrids Prepared by a Facile Synthesis Process and Their Magnetic Properties
}

\author{
Li Zhang ${ }^{1}$, Qing-Qing $\mathrm{Ni}^{2}{ }^{*}$, Toshiaki Natsuki ${ }^{1}$, Yaqin $\mathrm{Fu}^{2 \mathrm{a}}$ \\ ${ }^{1}$ Faculty of Textile Science \& Technology, Shinshu University, Japan \\ ${ }^{2 a}$ Key Laboratory of Advanced Textile Materials and Manufacturing Technology \\ Ministry of Education, Zhejiang Sci-Tech University, Hangzhou 310018, P.R. China \\ ${ }^{2 \mathrm{~b}}$ Faculty of Textile Science \& Technology, Shinshu University, Japan
}

\begin{abstract}
In this paper, a facile synthesis process is proposed to prepare multi-walled carbon nanotubes/magnetite (MWCNTs/ $/ \mathrm{Fe}_{3} \mathrm{O}_{4}$ ) hybrids. The process involves two steps: 1) water-soluble CNTs are synthesized by one-pot modification using potassium persulfate (KPS) as oxidant. 2) $\mathrm{Fe}_{3} \mathrm{O}_{4}$ is assembled along the treated CNTs by employing a facile hydrothermal process with the presence of hydrazine hydrate as the mineralizer. The treated CNTs can be easily dispersed in aqueous solvent. Moreover, X-ray photoelectron spectroscopy (XPS) analysis reveals that several functional groups such as potassium carboxylate $(-\mathrm{COOK})$, carbonyl $(-\mathrm{C}=\mathrm{O})$ and hydroxyl $(-\mathrm{C}-\mathrm{OH})$ groups are formed on the nanotube surfaces. The MWCNTs/ $/ \mathrm{Fe}_{3} \mathrm{O}_{4}$ hybrids are characterized with respect to crystal structure, morphology, element composition and magnetic property by x-ray diffraction (XRD), transmission electron microscopy (TEM), XPS and superconducting quantum interference device (SQUID) magnetometer. XRD and TEM results show that the $\mathrm{Fe}_{3} \mathrm{O}_{4}$ nanoparticles with diameter in the range of 20-60 nm were firmly assembled on the nanotube surface. The magnetic property investigation indicated that the $\mathrm{CNTs} / \mathrm{Fe}_{3} \mathrm{O}_{4}$ hybrids exhibit a ferromagnetic behavior and possess a saturation magnetization of $32.2 \mathrm{emu} / \mathrm{g}$. Further investigation indicates that the size of assembled $\mathrm{Fe}_{3} \mathrm{O}_{4}$ nanoparticles can be turned by varying experiment factors. Moreover, a probable growth mechanism for the preparation of $\mathrm{CNTs} / \mathrm{Fe}_{3} \mathrm{O}_{4}$ hybrids was discussed.
\end{abstract}

Keyword: carbon nanotubes; Carbon Nanotubes/Magnetite Hybrids; Magnetic Properties

\section{Introduction}

Carbon nanotubes (CNTs) have been widely applied as a promising material in many areas of nanoscience due to their outstanding physical and electrical properties such as high tensile strength, elastic modulus, excellent thermal and electrical

\footnotetext{
*Corresponding to: Qing-Qing Ni; E-mail: niqq@ shinshu-u.ac.jp Contract grant sponsor: Global COE Program and CLUSTER (the second stage) by the Ministry of Education, Culture, Sports, Science, and Technology, Japan.
} 
conductivity [1-3]. With the great progress achieved in this field, CNTs have been used to synthesize not only various polymer/CNTs composites [4-8] but also various CNTs/metal oxide hybrids [9-13], where CNTs can serve as high-performance supporting materials. The decoration of CNTs with metal oxide nanoparticles can give them new properties and potentials for various applications [14-18]. Among them, the CNTs coated $\mathrm{Fe}_{3} \mathrm{O}_{4}$ nanoparticles have attracted much increasing interest because of the excellent magnetic and biocompatible properties, which make them to be applied in electrochemical biosensing [19], biomanipulation [20], targeted drug delivery [21] and contaminant treatment [22]. A few efforts have been devoted to prepare hybrid materials of CNTs and $\mathrm{Fe}_{3} \mathrm{O}_{4}$ [23-28], however, surface modification of CNTs and subsequent assembly with synthesized $\mathrm{Fe}_{3} \mathrm{O}_{4}$ nanoparticles via covalent or noncovalent bonding is the most common preparation route. For example, Choi et al. prepared $\mathrm{CNTs} / \mathrm{Fe}_{3} \mathrm{O}_{4}$ hybrids based on the physically absorption between the PAAm- $\mathrm{Fe}_{3} \mathrm{O}_{4}$ nanoparticles and acids-treated CNTs [23]. And these methods often require complicated and time consuming operations. An alternative promising avenue to prepare $\mathrm{CNTs} / \mathrm{Fe}_{3} \mathrm{O}_{4}$ hybrids is in-situ synthesis methods such as high-temperature decomposition route [25] and solvothermal method [26-28]. However, these research groups often need costly raw materials and/or kinds of organic solvents.

This paper aims at reporting a cheap and green in-situ synthesis method to prepare $\mathrm{CNTs} / \mathrm{Fe}_{3} \mathrm{O}_{4}$ hybrids, which eliminates both harsh treatment conditions and the need for organic solvent. In the first step, we prepare water-soluble CNTs by one-step functionalization route which was designed by ourselves [29]. Actually, we introduce potassium carboxylate group $(-\mathrm{COOK})$ on the nanotube surfaces by mild oxidation process using potassium persulfate (KPS). It must be remembered that the most common way to generate carboxylic acid group $(-\mathrm{COOH})$ at nanotube surfaces consists of treating the CNTs under strong oxidizing conditions, which usually accompanies with a cutting process and deeply etching. Moreover, compared to carboxylic acid groups $(-\mathrm{COOH})$, the potassium carboxylate group $(-\mathrm{COOK})$ on nanotube surfaces is easier to be ionized in aqueous media, which causes the charged metal ions to attach to the nanotube surfaces easily. In the second step, we used these functionalized CNTs to prepare $\mathrm{CNTs} / \mathrm{Fe}_{3} \mathrm{O}_{4}$ hybrids by a hydrothermal process using ferrous sulfate hydrate $\left(\mathrm{FeSO}_{4} \cdot 7 \mathrm{H}_{2} \mathrm{O}\right)$ as a single iron precursor (with electromagnetic stirring). This facile and economical preparation route paves the way for wide potential application of $\mathrm{CNTs} / \mathrm{Fe}_{3} \mathrm{O}_{4}$ hybrids.

\section{Experimental}

\subsection{Materials}

All reagents were used of analytical grade without further purification. Multiwalled carbon nanotubes with a diameter of 20-30 nm, purchased from Wako Pure chemical Industries Ltd (Japan), were used as received.

\subsection{One-step functionalization of water-soluble MWNTs}

$80 \mathrm{mg}$ pristine MWNTs and $100 \mathrm{ml}$ deionized water were added to a flask and dispersed with the aid of an ultrasonic water bath for $60 \mathrm{~min}$ at room temperature. 
Then $0.9 \mathrm{~g}$ KPS was added to the flask and the $\mathrm{pH}$ of the reaction system was adjusted to 13 by adding concentrated $\mathrm{KOH}$ solution. The flask equipped with a reflux condenser and a magnetic stirring bar was kept at $85^{\circ} \mathrm{C}$ by vigorous mixing for $5 \mathrm{~h}$, and then cooled down to room temperature naturally. The contents of the flask were carefully diluted with water and dispersed with the aid of ultrasonic water bath, followed by a centrifugation process at $3000 \mathrm{rpm}$ for $5 \mathrm{~min}$. The centrifugation process was expected to separate the impurities and bundled MWNTs from the solution. The supernatant solution was collected and filtered through a hydrophilic polytetrafluoroethylene membrane $(47 \mathrm{~mm} / 0.45 \mu \mathrm{m}$, from Millipore) and washed with distilled water repeatedly. Finally, the products were dried overnight at $80^{\circ} \mathrm{C}$.

\subsection{Assembling $\mathrm{Fe}_{3} \mathrm{O}_{4}$ nanoparticals on water-soluble MWCNTs}

The assembling process of $\mathrm{Fe}_{3} \mathrm{O}_{4}$ on MWCNTs was carried out by a hydrothermal method according to ref [30] with some modification. In a typical procedure, water-soluble MWCNTs (30 mg) were dispersed into $100 \mathrm{ml}$ deionized water by sonication. Then $\mathrm{FeSO}_{4} \cdot 7 \mathrm{H}_{2} \mathrm{O}(86.9 \mathrm{mg})$ was added to the solution with stirring for 2 hours. After that, $\mathrm{N}_{2} \mathrm{H}_{4} \cdot \mathrm{H}_{2} \mathrm{O}(62.5 \mathrm{mg})$ were added with stirring for 5 min. The solution was transferred into high pressure microreactor MMJ-200 (Fig.1b, Omlab-tech. co., LTD, Japan). The microreactor was maintained at $150{ }^{\circ} \mathrm{C}$ for $8 \mathrm{~h}$ with stirring and cooled naturally to room temperature. The black products in the solution were collected after being rinsed with deionized water repeatedly and dried at $60{ }^{\circ} \mathrm{C}$ for $8 \mathrm{~h}$.

\subsection{Characterizations}

Transmission electron microscopy (TEM) and high-resolution transmission electron microscopic (HRTEM) images of the samples were observed through JEM-2010 electron microscope with an accelerating voltage of $200 \mathrm{kV}$. X-ray photoelectron spectroscopy (XPS) analyses were performed on a Kratos Axis Ultra DLD X-ray photoelectron spectrometer with a standard $\mathrm{Mg} \mathrm{K \alpha}(1256.6 \mathrm{eV}) \mathrm{X}$-ray source operated at $10 \mathrm{~mA}$ and $15 \mathrm{kV}$. All binding energies were referenced to $\mathrm{Au}$ $(4 \mathrm{f} 7 / 2)$ at $84 \mathrm{eV}$. Scanning electron microscopy (SEM) images of the products were observed using filed emission scanning electron microscopy (S-5000, Hitachi, Japan). The X-ray diffraction pattern (XRD) of the products was recorded on a Rigaku Geigerflex 2028 diffractometer (Rigaku, Japan) with $\mathrm{CuK} \alpha$ radiation (1.5418 ̊). The XRD data were collected over a $2 \theta$ range from 20 to $80^{\circ}$. Magnetic measurements were performed on a commercial superconducting quantum interference device (SQUID) magnetometer (Quantum Design, MPMS). Hysteresis loops were measured at $300 \mathrm{~K}$ in a magnetic field of in the range of $\pm 10 \mathrm{kOe}$.

\section{Results and discussion}

The pristine MWCNTs in our experiments were first treated by mild oxidation process using potassium persulfate (KPS). This one-step functionalization method is an effective method in preparing water-soluble MWCNTs by introducing the potassium carboxylate groups $(-\mathrm{COOK})$ on nanotube surfaces as well as purifying. By this process, treated CNTs can be easily dispersed in aqueous solvent, which can 
be readily confirmed by visual inspection (see Fig. 1c). The results show that the treated nanotubes form stable suspensions without visible aggregation even after one month. Figure 2 illustrates the typical scanning electron microscopy (SEM) images and transmission electron microscopy (TEM) images of MWCNTs before and after KPS treatment. SEM image of pristine CNTs (Fig. 2a) represents the partial bundled morphology and amorphous impurities. Comparison of treated CNTs (Fig. 2b) with pristine CNTs (Fig. 2a) indicates the obvious reduction of amorphous nanoparticles and bundled CNTs. Also this phenomenon can be observed from TEM images of pristine CNTs (Fig. 2c) and the treated CNTs (Fig. 2d). Moreover, the treated CNTs by KPS modification show no obvious shortening, which is different from ordinary treatment by concentrated acid treatment.

To determine the elements and the valence state of carbon presented in the treated nanotube surface, X-ray photoelectron spectroscopy (XPS) analysis was carried out. The typical XPS spectra are presented in Fig. 3a. Survey scans of functionalized MWCNTs show the presence of carbon, oxygen and potassium. Obviously, the high resolution scans of $\mathrm{C} 1 \mathrm{~s}$ of pristine MWCNTs and treated ones are quite different from each other (see the inset of Fig. 3a). In order to provide direct evidence for the added functional groups on the nanotube surfaces, the high resolution of $\mathrm{C} 1 \mathrm{~s}$ was analyzed with a fitting routine by decomposing $\mathrm{C} 1 \mathrm{~s}$ spectrum into individual mixed Gaussian-Lorentzian peaks after a Shirley background subtraction. The overall peak of $\mathrm{C} 1 \mathrm{~s}$ in the region of $282-292 \mathrm{eV}$ could be fitted by a superposition of four peaks (see Fig. 3b) , where the peaks of K $2 p$ at $293.2 \mathrm{eV}$ and $295.9 \mathrm{eV}$, effecting $\mathrm{C} 1 \mathrm{~s}$ spectrum, should be taken into account during curve fitting. The main binding energy peak $(284.4 \mathrm{eV})$ is attributed to the $\mathrm{C}-\mathrm{C} 1 \mathrm{~s}$, while the other three peaks are assigned to $-\mathrm{C}-\mathrm{OH}(286.2 \mathrm{eV}),-\mathrm{C}=\mathrm{O}(287.6 \mathrm{eV})$, and $-\mathrm{COOK}(288.9$ $\mathrm{eV}$ ), respectively [31]. According to Fig. 3b, we can quantitatively calculate the content of potassium carboxylate group (about $8.5 \%$ ) based on the ratios of peak areas.

Hydrothermal method has sparked much interest in recent years, due to its operational simplicity, and capability for large-scale production [32-35]. After the hydrothermal process of assembling the functionalized MWCNTs with $\mathrm{Fe}_{3} \mathrm{O}_{4}$, powder X-ray diffraction patterns (XRD) of the water-soluble CNTs and products were recorded (see Fig. 4). The XRD result shows that the product was a mixture of two phases, cubic $\mathrm{Fe}_{3} \mathrm{O}_{4}$ and MWCNTs. The diffraction peak at $26^{\circ}$ can be indexed to (002) reflection of the MWCNTs (see the inset of Fig. 4), similar to the result in the literature [28]. All other diffraction peaks can be well indexed to the magnetic cubic structure of $\mathrm{Fe}_{3} \mathrm{O}_{4}$ (JCPDS 79-0419) with lattice constants of $\alpha=8.396 \AA$. And no other peaks due to the hematite were detected in the XRD patterns, indicating the formation of the pure magnetic products. The strong and sharp diffraction peaks reveal the well crystallization of the product.

The structure and morphology of the obtained products were investigated by TEM. Figure 5a gives the representative TEM image of the products, which indicates that the MWCNTs were assembled with magnetite nanoparticles, and the diameters of nanoparticle are in the range of 20-60 $\mathrm{nm}$. Although the products had been sonicated 
in ethanol before TEM measurements, a great amount of magnetite nanoparticles are still found on the nanotube surface and this indicates the strong interaction between MWCNTs and magnetite nanoparticles. More detailed structural information of the products was provided by the high-resolution TEM (HRTEM) analysis, and Fig. 5b shows the HRTEM image taken from an individual hybrid structure. Clear lattice fringes are observed in HRTEM image, indicting that crystalline particles formed in the hydrothermal process. Moreover, the measured spacing of the crystallographic planes is about $0.48 \mathrm{~nm}$, which is close to that of the (111) lattice planes of magnetite crystals. In order to examine the relationship between the mixing process and the formed nanoparticle size during the hydrothermal process, $\mathrm{Fe}_{3} \mathrm{O}_{4}$ nanoparticles were prepared with and without electromagnetic mixing. Fig. 5c (the synthesis process with mixing) clearly shows that the sizes of $\mathrm{Fe}_{3} \mathrm{O}_{4}$ particles are well controlled in the range of 20-70 $\mathrm{nm}$. However, when the hydrothermal process without mixing, the particle sizes are very difficult to manipulate (see Fig. 5d). Therefore, in our system, the electromagnetic mixing are crucial to control the size of $\mathrm{Fe}_{3} \mathrm{O}_{4}$ nanoparticle assembled on the nanotube surface.

X-ray photoelectron spectroscopy (XPS) was used to explore the surface chemistry of the MWCNTs/ $/ \mathrm{Fe}_{3} \mathrm{O}_{4}$ hybrids. The typical survey spectrum of the products (see Fig. 6) shows the presence of Fe, O, N and C. The high resolution Fe $2 p$ spectrum (see the inset of Fig. 6) shows that the binding energies of the Fe $2 \mathrm{p}_{3 / 2}$ and Fe $2 p_{1 / 2}$ locates at $711.2 \mathrm{eV}$ and $725.0 \mathrm{eV}$, respectively. The results agree with literature data for magnetite [36,37], thus substantiating the XRD result. Moreover, the $\mathrm{Fe} / \mathrm{O}$ atomic ratio is about $3 / 4$ based on the ratio of the peak areas and relative sensitivity factor.

In accordance with these results, a possible mechanism leading to $\mathrm{CNTs} / \mathrm{Fe}_{3} \mathrm{O}_{4}$ hybrids is proposed in Fig. 1a. After modification by KPS, the potassium carboxylate groups $(-\mathrm{COOK})$ were formed on the nanotube surfaces, which makes the treated CNTs disperse easier in water with a stable manner. It is obvious that the negatively charged functional groups such as carboxylic anion $\left(-\mathrm{COO}^{-}\right)$on the nanotube surfaces can absorb positive charge $\mathrm{Fe}^{2+}$ species, which may act as the nucleation center. Those nuclei could initiate the growth of $\mathrm{Fe}_{3} \mathrm{O}_{4}$ nanoparticles, and then attach tightly to nanotube surfaces. In alkalescence medium, hydrazine hydrate can serve as either an oxidant or a reducer $[30,38]$,

As an oxidant:

$$
2 \mathrm{NH}_{3}+2 \mathrm{OH}^{-} \longleftrightarrow \mathrm{N}_{2} \mathrm{H}_{4}+2 \mathrm{H}_{2} \mathrm{O}+2 \mathrm{e}, \phi^{0}=0.1 \mathrm{~V}
$$

As a reducer:

$$
\mathrm{N}_{2} \mathrm{H}_{4}+4 \mathrm{OH}^{-} \longleftrightarrow \mathrm{N}_{2}+4 \mathrm{H}_{2} \mathrm{O}+4 \mathrm{e}, \phi^{0}=0.15 \mathrm{~V}
$$

Where $\phi^{0}$ is criterion electromotance. Thus, in the hydrothermal process, some of $\mathrm{Fe}^{2+}$ would be in situ oxidized into $\mathrm{Fe}^{3+}$ and then coprecipitated into $\mathrm{Fe}_{3} \mathrm{O}_{4}$ crystallites, which would be anchored to the CNTs. The probable chemical mechanism for the hydrothermal formation of the $\mathrm{Fe}_{3} \mathrm{O}_{4}$ nanoparticles assembled on CNTs can be 
expressed as follow:

$$
2 \mathrm{Fe}^{3+}+\mathrm{Fe}^{2+}+8 \mathrm{OH}^{-} \longrightarrow \mathrm{Fe}_{3} \mathrm{O}_{4} \downarrow+4 \mathrm{H}_{2} \mathrm{O}
$$

Generally, the driving force for oriented aggregation of nanocrystals is attributed to the tendency to decrease the surface energy by self-organization. In our case, the assembling process of the $\mathrm{Fe}_{3} \mathrm{O}_{4}$ nanocrystals into nanoparticles is also contributed to oriented aggregation. Moreover, the size of assembled nanoparticles can be turned by varying experiment factors, such as the weight ratio of MWCNTs and iron precursor, and stirring speed during hydrothermal process.

As a magnetic material, the magnetic property of the $\mathrm{CNTs} / \mathrm{Fe}_{3} \mathrm{O}_{4}$ hybrids is important to its applications. The magnetization curves at $300 \mathrm{~K}$ of the $\mathrm{CNTs} / \mathrm{Fe}_{3} \mathrm{O}_{4}$ hybrids are shown in Fig. 7a. The results show that the products have saturation magnetization of $32.2 \mathrm{emu} / \mathrm{g}$, which is lower than that of the corresponding bulk (92 $\mathrm{emu} / \mathrm{g}$, [39]) resulting from the existence of MWCNTs. And the coercivity is about 80 Oe at $300 \mathrm{~K}$ (see the inset of Fig. 7a). Therefore, the products show a soft ferromagnetic behavior, which is the same as $\mathrm{Fe}_{3} \mathrm{O}_{4}$. The separability of the products was tested by holding a commercial magnet close to the sample as shown in Fig. $7 \mathrm{~b}$. The black products were attracted toward the magnetite in a short period, indicating that it is possible to manipulate the products by an external magnetic field.

\section{Conclusion}

Water-soluble multiwalled carbon nanotubes (MWCNTs) were synthesized by one-pot modification using KPS. Based on water-soluble CNTs, the MWCNTs/ $/ \mathrm{Fe}_{3} \mathrm{O}_{4}$ hybrids were successfully prepared by a facile hydrothermal process with electromagnetic stirring. For water-soluble CNTs, the effectiveness of modification route was confirmed by FESEM, TEM and XPS. For the CNTs/ $\mathrm{Fe}_{3} \mathrm{O}_{4}$ hybrids, the XRD, TEM and XPS results revealed that the MWCNTs were assembled with magnetite nanoparticles, and the diameters of nanoparticle in the range of 20-60 nm. The SQUID result shows that the MWCNTs/ $/ \mathrm{Fe}_{3} \mathrm{O}_{4}$ hybrids exhibit a ferromagnetic behavior and possess a higher coercivity. Further investigation shows that the size of assembled $\mathrm{Fe}_{3} \mathrm{O}_{4}$ nanoparticles can be turned by varying experiment factors.

\section{Acknowledgements}

This work was partly supported by Grant-in-Aid for Global COE Program and CLUSTER (the second stage) by the Ministry of Education, Culture, Sports, Science, and Technology, Japan.

\section{References}

[1] R.H. Baughman, N.A. Zakhidov, W.A. de Heer, Science 297 (2002) 787.

[2] D. Tasis, N. Tagmatarchis, A.Bianco, M. Prato, Chem. Rev. 106 (2006) 1105.

[3] J.N. Coleman, U. Khan, W.J. Blau, Y.K. Gun'ko, Carbon 44 (2006) 1624. 
[4] L. Ci, J. Suhr, V. Pushparaj, X. Zhang, P.M. Ajayan, Nano. Lett. 8 (2008) 2762.

[5] Y.K. Choi, K.I. Sugimoto, S.M. Song, Y. Gotoh, Y. Ohkoshi, M. Endo, Carbon 43 (2005) 2199.

[6] Y.T. Shieh, G.L. Liu, K.C. Hwang, C.C. Chen, Polymer 46 (2005) 10945.

[7] G.L. Hwang, Y.T. Shieh, K.C. Hwang, Adv. Funct. Mater. 18 (2004) 487.

[8] E. Bekyarova, E.T. Thostenson, A. Yu, H. Kim., J. Gao, J. Tang, H.T. Hahn, T.W.

Chou, M.E. Itkis, R.C. Haddon, Langmuir 23 (2007) 3970.

[9] Z. Liu, B. Han, Adv Mater 21 (2009) 825.

[10] C. Li, N. Sun, J. Ni, J. Wang, H. Chu, H. Zhou, M. Li, Y. Li, J. Solid State Chem. 181 (2008) 2620.

[11] N. Du, H. Zhang, B. Chen, J. Wu, X. Ma, Z. Liu, Y. Zhang, D. Yang, X. Huang, J. Tu, Adv. Mater. 19 (2007) 4505.

[12] G. Korneva, H. Ye, Y. Gogotsi, D. Halverson, G. Friedman, J.C. Bradley, K.G. Kornev, Nano. Lett. 5 (2005) 879.

[13] R. Zhang, X. Wang, Chem. Mater. 19 (2007) 976.

[14] D.R. Kauffman, A. Star, Angew. Chem. Int. Ed. 47 (2008) 6550.

[15] B. Yu, F. Zhou, G. Liu, Y. Liang, W.T.S. Huck, W. Liu, Chem. Commun. 22 (2006) 2356.

[16] X. Wang, B. Xia, X. Zhu, J. Chen, S. Qiu, J. Li, J. Solid State Chem. 181 (2008) 822.

[17] N. Du, H. Zhang, B. Chen, X. Ma, Z. Liu, J. Wu, D. Yang, Adv. Mater. 19 (2007) 1641.

[18] Z. Liu, J. Wang, D. Xie, G. Chen, Small 4 (2008) 462.

[19] C. Gao, W. Li, H. Morimoto, Y. Nagaoka, T. Maekawa, J. Phys. Chem. B 110 (2006) 7213.

[20] F. Yang, D.L. Fu, J. Long, Q.X. Ni, Med. Hypotheses 70 (2008) 765.

[21] X. Peng, Z. Luan, Z. Di, Z. Zhang, C. Zhu, Carbon 43 (2005) 855.

[22] P. Xu, D. Cui, B. Pan, F. Gao, R. He, Q. Li, T. Huang, C. Bao, H. Yang, Appl. Surf. Sci. 254 (2008) 5236.

[23] W.S. Ko, M.S. Yang, H.J. Choi, Mater. Lett. 63 (2009) 861.

[24] L. Jiang, L. Gao, J. Electroceram 17 (2006) 87.

[25] J. Wan, W. Cai, J. Feng, X. Meng, E. Liu, J. Mater. Chem. 17 (2007) 1188.

[26] L. Jiang, L. Gao, Chem. Mater. 15 (2003) 2848.

[27] B. Jia, L. Gao, J. Phys. Chem. B 111 (2007) 5337.

[28] B. Jia, L. Gao, J. Sun, Carbon 45 (2007) 1476.

[29] L. Zhang, Q,Q, Ni, Y. Fu, T. Natsuki, Appl. Surf. Sci. 25 (2009) 7095.

[30] H. Zhu, D. Yang, L. Zhu, Surf. Coat. Tech. 201 (2007) 5870.

[31] N. Kovtyukhova, E. Buzaneva, A. Senkevich, Carbon 36 (1998) 549.

[32] L. Chen, Y. Shen, J. Bai, Mater. Lett. 63 (2009) 1099.

[33] M.A. Dar, Q. Ahsanulhaq, Y.S. Kim, J.M. Sohn, W.B. Kim, H.S. Shin, Appl. Surf. Sci. 255 (2009) 6279.

[34] H. Zhu, D. Yang, G. Yu, H. Zhang, D. Jin, K. Yao, J. Phys. Chem. B 110 (2006) 7631.

[35] L. Chen, C. Zhao, J. Bai, Chem. Lett. 38 (2009) 276. 
[36] Y. Sahoo, A. Goodarzi, M.T. Swihart, T.Y. Ohulchanskyy, N. Kaur, E.P. Furlani, P.N. Prasas, J. Phys. Chem. B 109 (2005) 3879.

[37] Z. Qian, Z. Zhang, Y. Chen, J. Colloid Interf. Sci. 327 (2008) 354.

[38] J. Wang, J. Sun, Q. Sun, Q. Chen, Mater. Res. Bull. 38 (2003) 1113.

[39] D.H. Han, J.P. Wang, H.L. Luo, J. Magn. Magn. Mater. 136 (1994) 176. 


\section{Figure captions}

Figure 1. (a) Schematic illustration of preparation of $\mathrm{CNTs} / \mathrm{Fe}_{3} \mathrm{O}_{4}$ hybrids; (b) The photograph of high pressure microreactor MMJ-200; (c) The water-solubility of pristine CNTs and treated CNTs.

Figure 2. FESEM images of (a) pristine CNTs and (b) treated CNTs; TEM images of (c) pristine CNTs and (d) treated CNTs.

Figure 3. (a) XPS spectra of pristine CNTs and treated CNTs (the inset shows the high-resolution scan of $\mathrm{C} 1 \mathrm{~s}$ region); (b) The curve fitting of the carbon $1 \mathrm{~s}$ of treated CNTs.

Figure 4. XRD pattern of $\mathrm{CNTs} / \mathrm{Fe}_{3} \mathrm{O}_{4}$ hybrids (the inset shows the XRD pattern of treated CNTs).

Figure 5. (a) Typical TEM image of $\mathrm{CNTs} / \mathrm{Fe}_{3} \mathrm{O}_{4}$ hybrids; (b) The HRTEM image of individual hybrid structure; (c) The TEM images of $\mathrm{Fe}_{3} \mathrm{O}_{4}$ nanoparticles by hydrothermal process with electromagnetic mixing; (d) The TEM images of $\mathrm{Fe}_{3} \mathrm{O}_{4}$ nanoparticles by hydrothermal process without mixing.

Figure 6. XPS spectrum of $\mathrm{CNTs} / \mathrm{Fe}_{3} \mathrm{O}_{4}$ hybrids (the inset shows the high-resolution scan of Fe $2 p$ region).

Figure 7. (a) Hysteresis loops of the $\mathrm{CNTs} / \mathrm{Fe}_{3} \mathrm{O}_{4}$ hybrids measured at $300 \mathrm{~K}$; (b) Separation $\mathrm{CNTs} / \mathrm{Fe}_{3} \mathrm{O}_{4}$ hybrids from solution under an external magnetic field. 


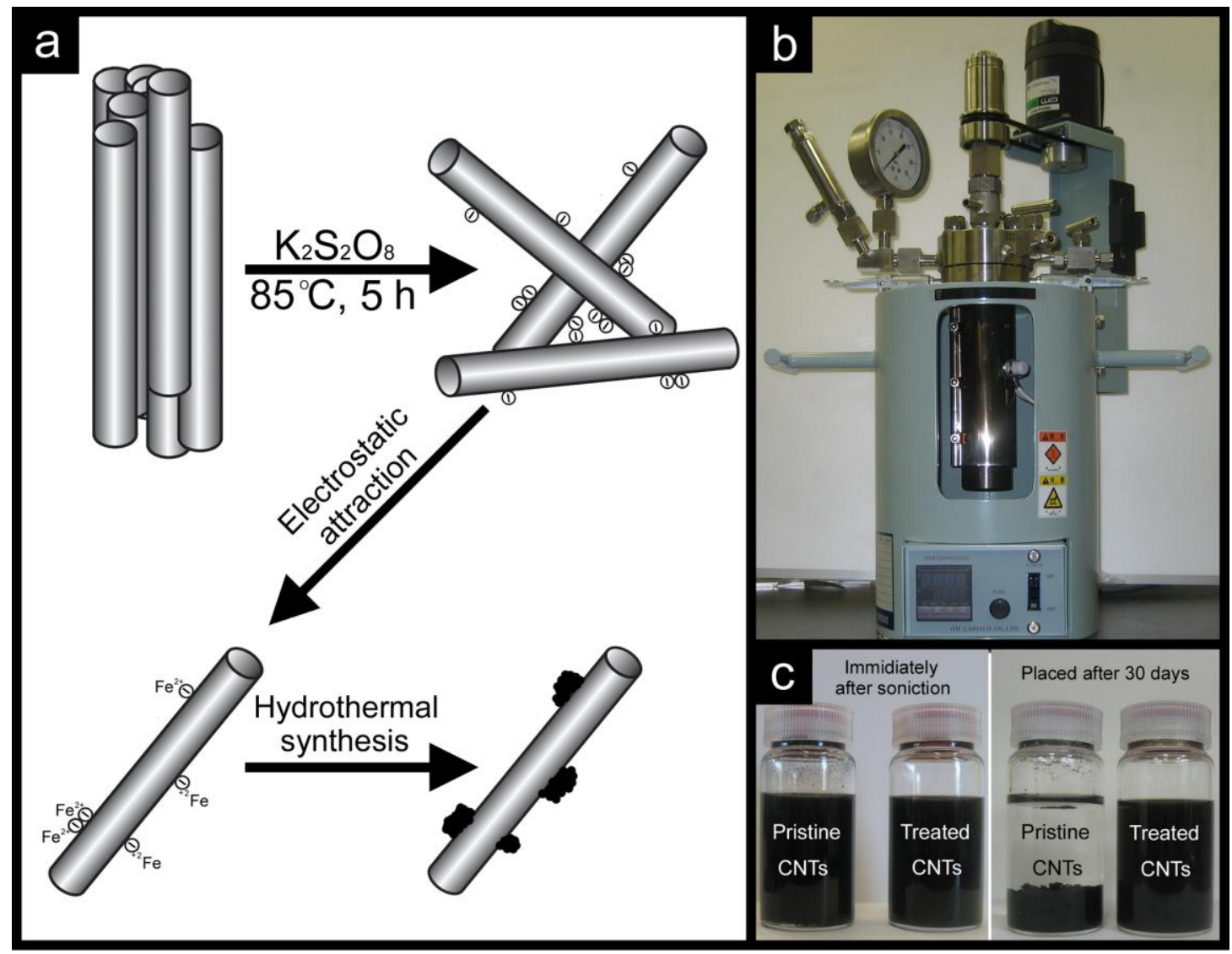

Figure 1. 

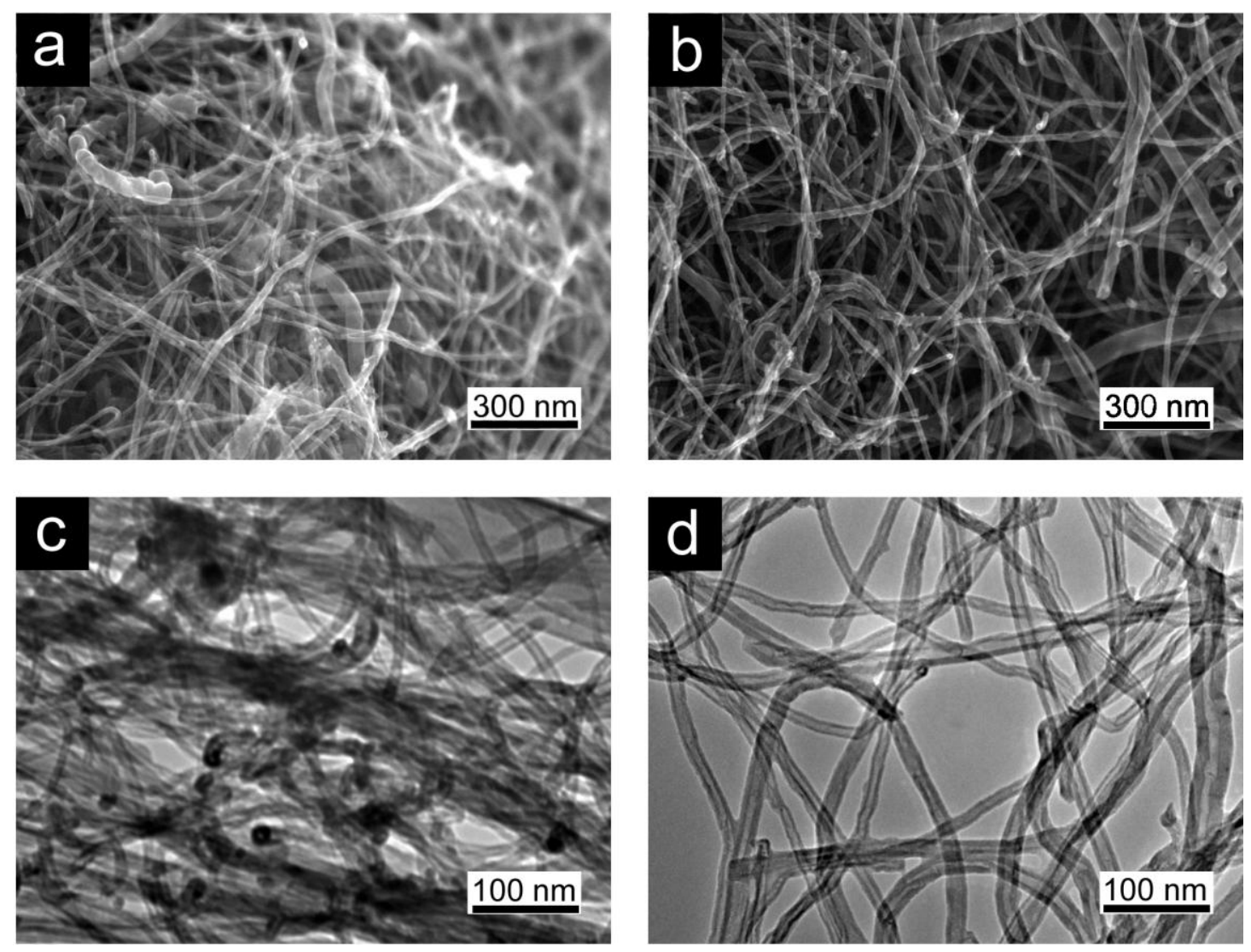

Figure 2. 

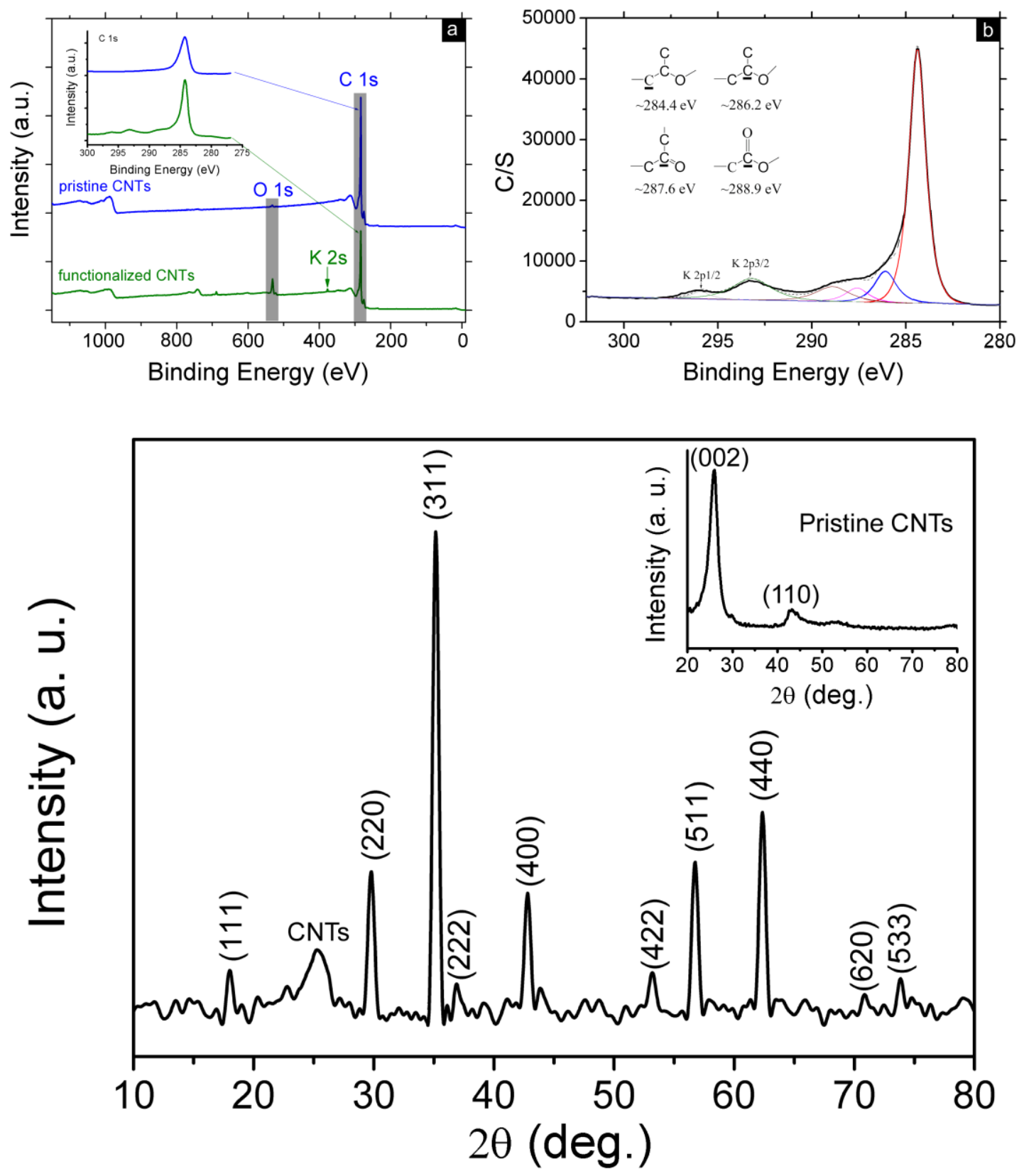

Figure 3. 


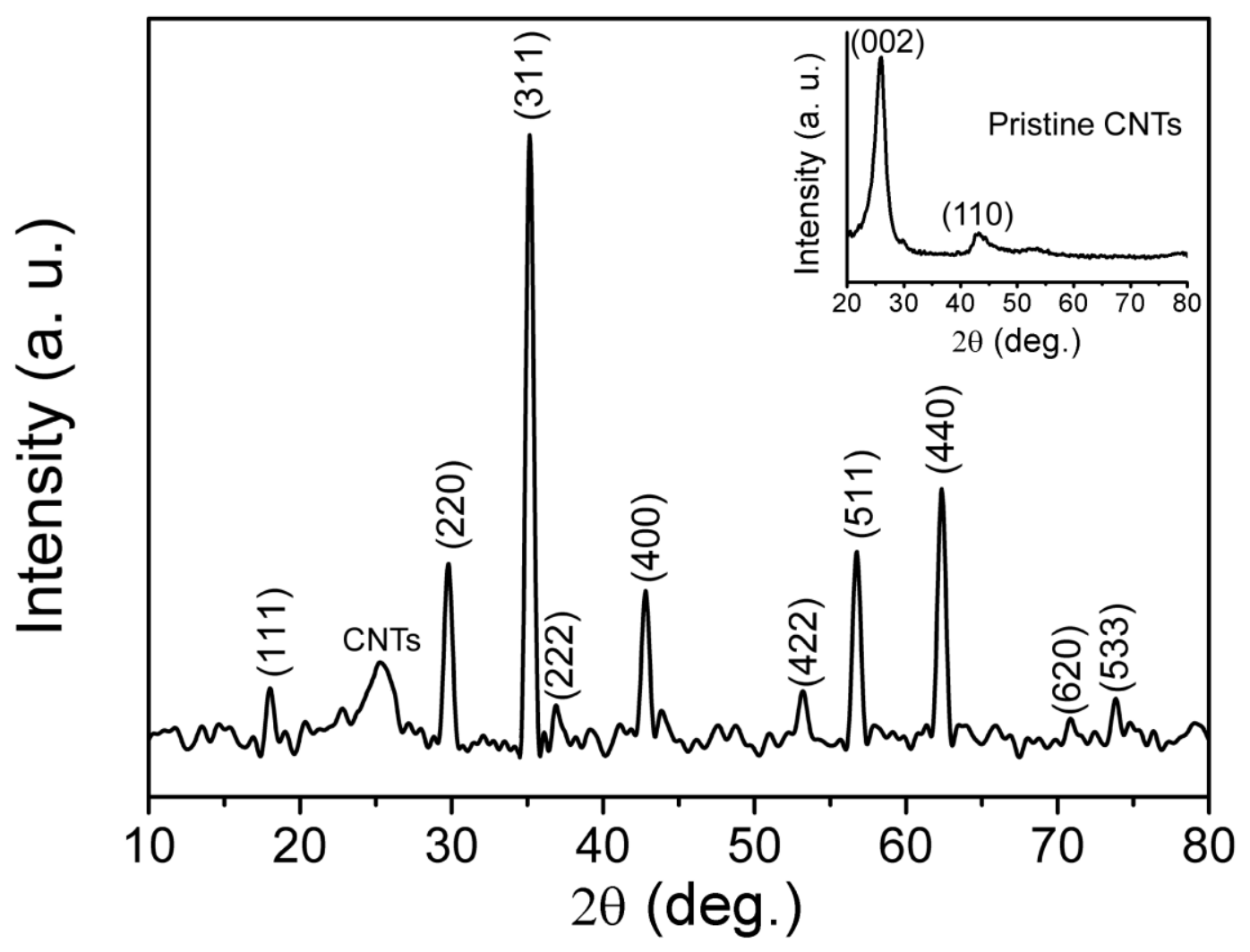

Figure 4. 

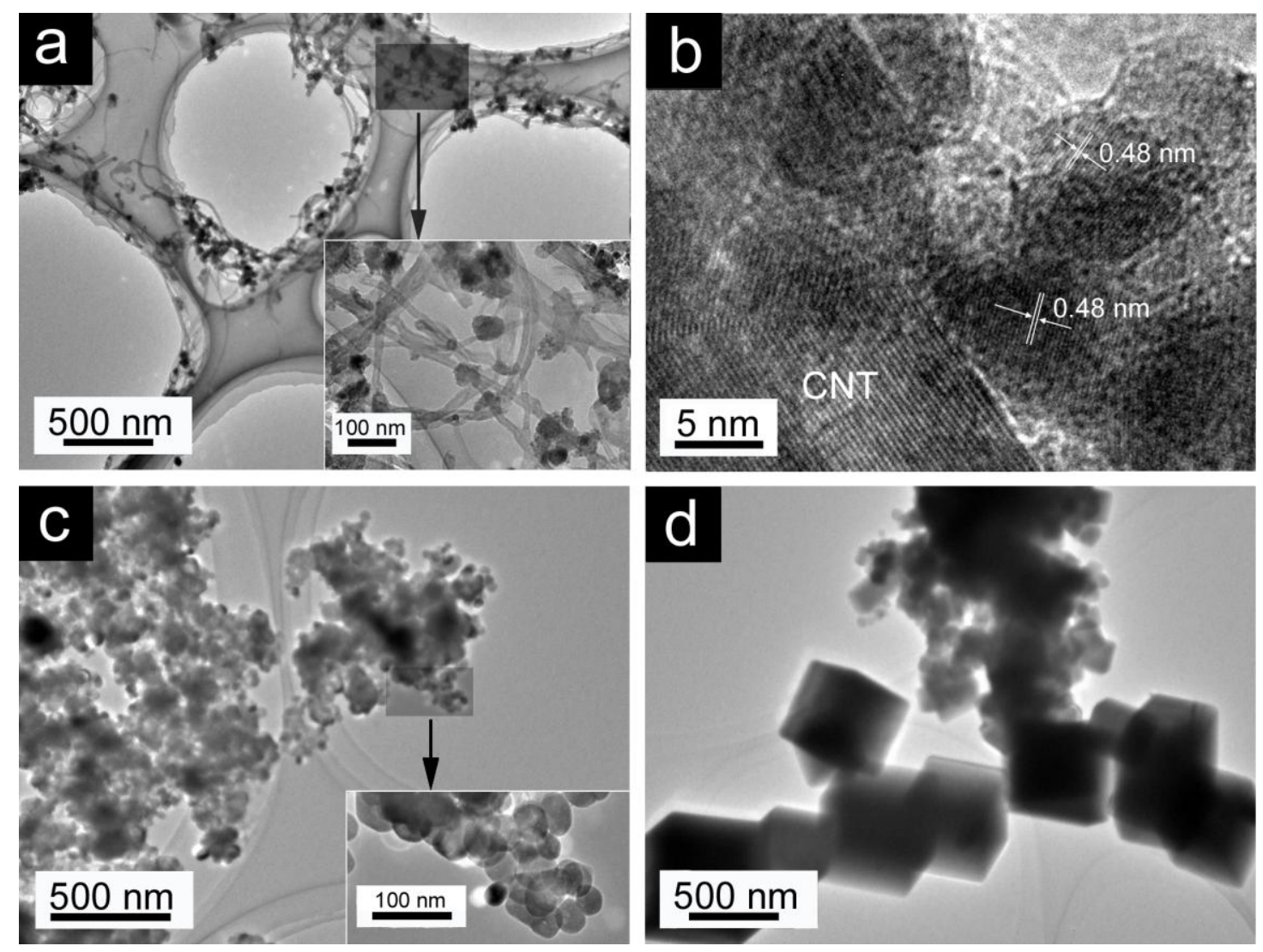

Figure 5. 


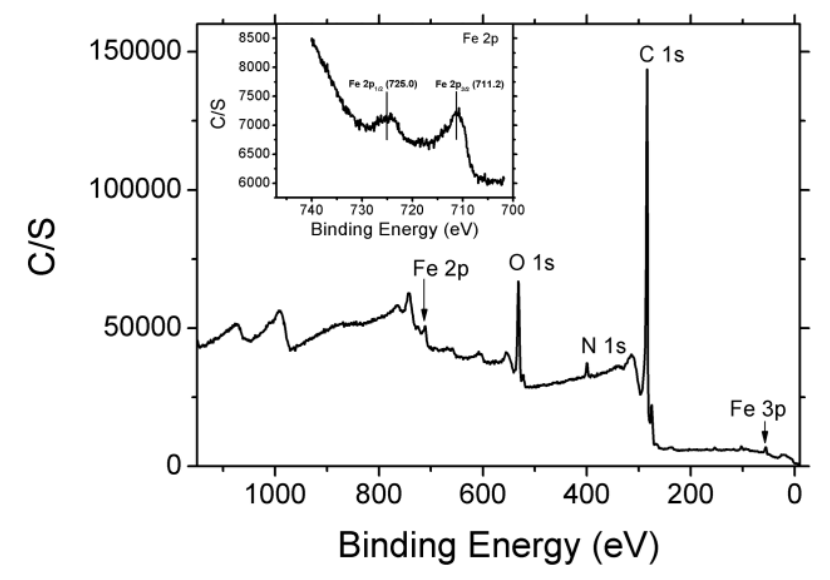

Figure 6. 

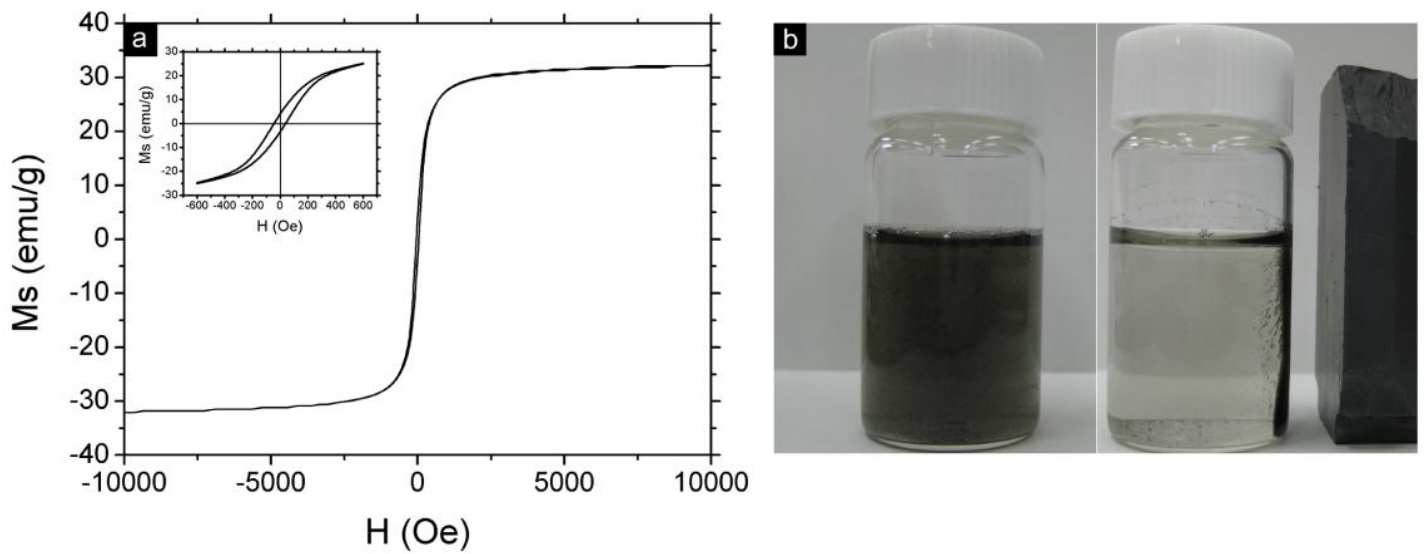

Figure 7. 